\title{
Review of EEG feature selection by neural networks
}

\author{
Ildar Rakhmatulin
}

\begin{abstract}
:
The basis of the work of electroencephalography (EEG) is the registration of electrical impulses from the brain using a special sensor or electrode. This method is used to treat and diagnose various diseases. In the past few years, due to the development of neural network technologies, the interest of researchers in EEG has noticeably increased. Neural networks for training the model require obtaining data with minimal noise distortion. In the processing of EEG signals to eliminate noise (artifacts), signal filtering and various methods for extracting signs are used. The presented manuscript provides a detailed analysis of modern methods for extracting the signs of an EEG signal used in studies of the last decade. The information presented in this paper will allow researchers to understand how to more carefully process the data of EEG signals before using neural networks to classify the signal. Due to the absence of any standards in the method of extracting EEG signs, the most important moment of this manuscript is a detailed description of the necessary steps for recognizing artifacts, which will allow researchers to maximize the potential of neural networks in the tasks of classifying EEG signal.
\end{abstract}

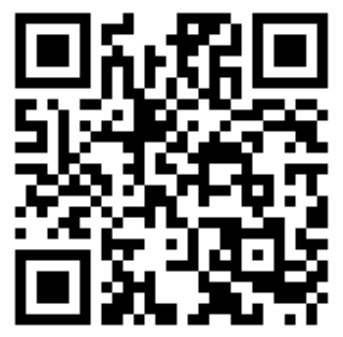

IJSB

Accepted 10 August 2020 Published 17August 2020 DOI: 10.5281/zenodo.3987894

Keywords: Fast Fourier transform (FFT) EEG, wavelet transform (WT) EEG, EEG machine learning, extraction of EEG features, EEG signal filtering, EEG neural networks, bci feature selection, brain computer interface

Rakhmatulin Ildar, PhD. South Ural State University, Department of Power Plants Networks and Systems, Chelyabinsk city, Russia, 454080. 


\section{Introduction}

Feature extraction is the process of extracting useful information from an electroencephalogram (EEG) signal to represent the correct data set before performing the classification procedure. Over the past decade, the database of the US National Library of Medicine, the popular libraries of Elsevier, Taylor \& Francis, ieeexplore.ieee.org, more than 500 works dedicated to the allocation of processing of EEG signals were presented. The analysis of these works allows us to conclude that the surge of interest in this topic is since it became possible to use various mxodels of neural networks as a classification tool. These researches focus on the signal EEG classification stage. The choice of the electrode does not carry great difficulties, due to the simplicity of the criterion for its selection (impedance, noise, etc.), but the competent implementation of the signs carry certain difficulties. For models of neural networks engaged in the tasks of signal classification, the main criterion for the correct operation is the correct receipt of a dataset. Therefore, the purpose of this manuscript is to conduct a detailed analysis of modern methods for extracting features of EEG signals. This study is especially relevant when identifying features for use in classification problems using machine learning. The following methods for extracting features were considered by Al-Fahoum (2014): temporal frequency distributions (TFD), fast Fourier transform (FFT), eigenvector methods (EM), wavelet transform (WT) and autoregressive method (ARM). In the paper, the characteristics of various methods were briefly compared. Mane et al. (2019), similarly, as and author in the article described above, various methods of feature extraction are compared, such as wavelet transform, independent component analysis, principal component analysis, autoregressive model, and empirical mode decomposition. Raut (2010) in his paper considered three subsets of the obtained characteristics by the method of extracting tracks, wavelet transform, and fractional Fourier transform. The author carried a comparison of efficiency out by classification using reference vector machines.

Despite the stated goals, the described works did not fully reveal the potential and significance of the feature extraction technique in classification problems by machine learning. The main disadvantage:

- these works describe several methods for extracting attributes and testing them on one of the models of neural networks, that, according to the author, does not yet prove the correctness of the operation for extracting features. Since it is necessary to use several neural networks for verification;

- the recommendations are not presented in the works on the correct extraction of features in the tasks of classifying EEG signals for subsequent studies.

This manuscript will try to avoid these shortcomings and fully disclose a method for extracting features from EEG signals. The algorithm for interpreting EEG signals consists of 4 steps, figure 1.

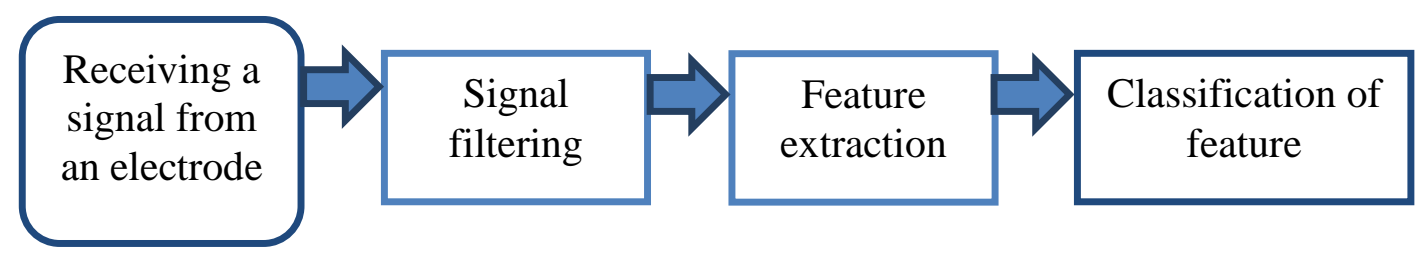

Fig.1. Algorithm for interpreting EEG signals

In this paper, we will consider in detail the 2nd and 3rd steps, which have different tools for implementing but the same purpose - the extraction of features from the signal.

\section{International Journal of Science and Business Published By Email: editor@ijsab.com Website: ijsab.com




\section{Filtering the EEG signal}

Filtering is necessary to attenuate certain frequency components of the signal. When working with EEG, this is especially important. Are known are 4 frequency bands of human EEG waves (pic. 1, a):

- Delta: $3 \mathrm{~Hz}$ or lower. Delta waves are the slowest wave and have the largest amplitude. Usually, at the 3rd and 4th stages of sleep and young children of the first year, this signal band is dominant;

- Theta: with a frequency range of $3.5 \mathrm{~Hz}$ to $7.5 \mathrm{~Hz}$. This signal is noticeable in children under 13 years of age;

- Alpha: frequency range from 7.5 to $13 \mathrm{~Hz}$. Typically, the posterior regions of the head emit alpha signals. This is the main type of signal in a normal adult in a relaxed state;

- Beta: frequency greater than $14 \mathrm{~Hz}$. It is markedly distinguished in patients who are alert for a long time or worry.

Based on this, we can conclude about what frequency signals should be reduced. As a noise filter implementation, both filters made on analog elements and digital can be used. There are a huge number of schemes for implementing filters; their consideration is not included in the tasks of this manuscript.

Mathematically, filters can be implemented in the following way. Let the original EEG signal X contain data obtained from $M$ leads (channels). Moreover, in each lead $m \in\{1,2, \ldots, M\}$ the signal is presented as a set of discrete samples taken at time instants with numbers $n=0,1, \ldots$, $\mathrm{N}-1$, where $\mathrm{N}$ is the total number of samples. Then in vector form, the signal can be represented as:

$X=(\bar{x}(0), \ldots, \bar{x}(n), \ldots, \bar{x}(N-1))=\left(\begin{array}{ccc}x_{1}(0) & \cdots & x_{1}(N-1) \\ \vdots & \ddots & \vdots \\ x_{M}(0) & \cdots & x_{M}(N-1)\end{array}\right)$,

where $\bar{x}(n)=\left(x_{1}(n), \ldots, x_{m}(n), \ldots, x_{m}(n)\right)^{T}$ - signal value at time with number $n \in\{1,2, \ldots, M\} ; x_{m}(n)$ - the value of the $m$-th signal component at a specified point in time, $m \in\{1,2, \ldots, M\}$

To filter a signal represented as a sequence of discrete samples, discrete filters are used, which in the general case can be described by a difference equation:

$y(n)=\sum_{i=0}^{P} b_{i} x(n-i)-\sum_{k=1}^{Q} a_{k} y(n-k), n \in\{0,1, \ldots, N-1\}$,

where $y(n)$ - sample of the output signal at the current moment of time $n, x(n-i)$ - samples of the input signal at previous times $(n-i), n \in\{0,1, \ldots, N-1\}, y(n-k)$ - samples of the output the signal at previous times (n-k), $n \in\{1,2, \ldots Q\}$, $b_{i}-$ coefficients of the input signal, $a_{k}-$ feedback coefficients, $\mathrm{P}$ - order of the input signal, $\mathrm{Q}$ - feedback order.

The main characteristic of the filter is its transfer function $\mathrm{H}(\mathrm{z})$, which for discrete systems is determined using the Z-transform as follows:

$H(z)=\frac{y(z)}{x(z)}=\frac{\sum_{i=1}^{P} b_{i} z^{-i}}{1+\sum_{k=1}^{Q} a_{i} z^{-k}}$,

where $y(z), x(z)$ are the Z-transformations for the signals $y(n), x(n)$, respectively, $f \in[0,1]$ is the relative signal frequency (the ratio of the signal frequency to the sampling frequency).

A discrete filter that is used as an input is called a recursive filter or an infinite impulse response filter (IIR). Examples of such filter filters are Butterworth, Chebyshev, Bessel filters and elliptical filters. A discrete filter without feedback is called a transverse filter or a filter with a finite impulse response (FIR). One of the features of FIR filters is their linear phase response in the passband, which provides the same group delay at different frequencies.

\section{International Journal of Science and Business} Email: editor@ijsab.com Website: ijsab.com 
Figure 2 showed the result of the process of filtering the EEG signal from the interference of a $50 \mathrm{~Hz}$ network.
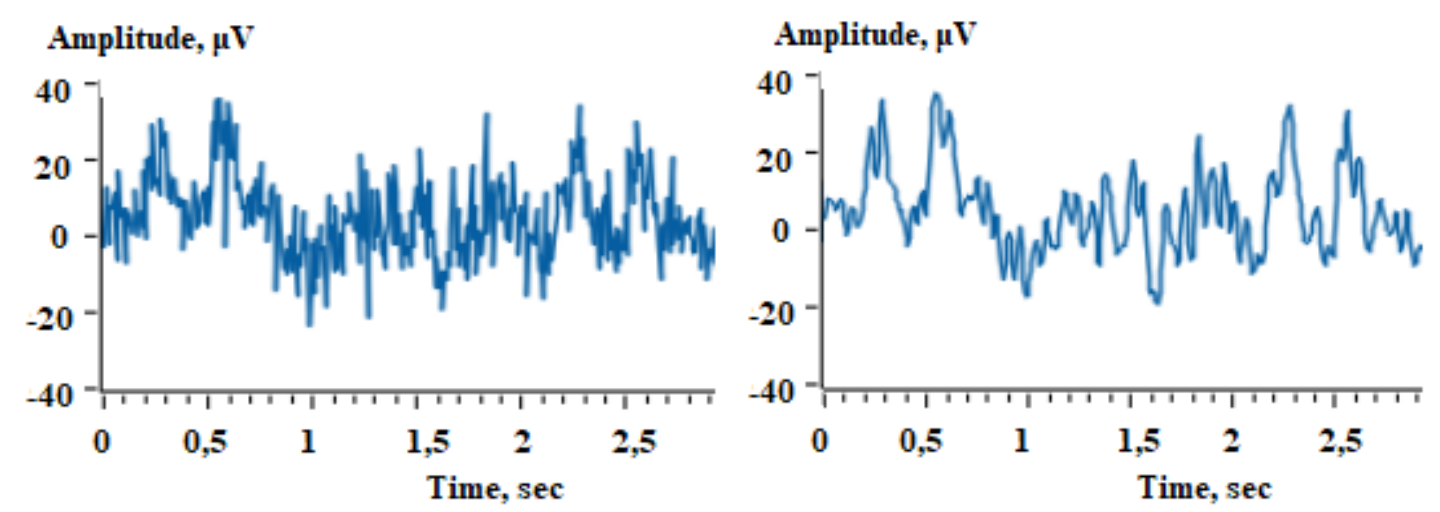

Fig.2. The result of the filtering process of the EEG signal from the interference of the network $50 \mathrm{~Hz}$.

In this case, the interference in the EEG signal from the network is a good example of the fact that neural networks that are more sensitive to artifacts are highly dependent on the process of filtering the signal. The neurophysiologist will be able to identify this artifact without software processing, while in the neural network this noise will introduce a significant error.

\section{EEG feature extraction}

There are many different methods for performing feature extraction. But at the same time, the analysis shows that approximately $85 \%$ of the work on this topic involved the Fourier transform and Wavelet transform. There are many different methods for performing feature extraction. But at the same time, the analysis shows that approximately 85\% of the work on this topic involved the Fourier transform and Wavelet transform. Many researches, using new methods for feature extract, for comparing use the Fourier decomposition method as a reference one.

\subsection{EEG Fourier Transform}

The Fourier transform is a family of mathematical methods based on the decomposition of the initial continuous function of time into a set of basic harmonic functions (which are sinusoidal functions) of different frequency, amplitude and phase.

The main idea of the transformation is that any function can be represented as an infinite sum of sinusoids, each of which will be characterized by its amplitude, frequency and initial phase. Al-Salman et al. (2019) and Hyvärinen et al. (2010) and Sitnikova (2009) and Chen (2014) and Kato et al. (2018) showed in detail that the continuous Fourier transform and the discrete Fourier transform have not found wide application in the process of extracting attributes due to their low efficiency. The most popular is the decomposition of the signal into harmonic components using the Fast Fourier transform. For the signal $x(n)$, presented in the form of a sequence of samples (fig. 3, up), taken with sampling frequency Fs, time moments with numbers $n=0,1, N-1$, the discrete Fourier transform is defined as:

$F(k)=\sum_{n=0}^{N-1} x(n) * e^{-\frac{2 \pi j}{N} k n}, k=0,1, \ldots, N-1$,

where $F(k)$ is the complex amplitude of the sinusoidal signal with a frequency $k^{*} \Delta \mathrm{f}, \Delta \mathrm{f}=\mathrm{Fs}$ / $\mathrm{N}$ resolution (step) in frequency, $\mathrm{x}(\mathrm{n})$ are the measured signal values at time instants with numbers $\mathrm{n}=0,1, \ldots . \mathrm{N}-1$.

The implementation of the Fast Fourier transform is presented in figure 3.

\section{International Journal of Science and Business Email: editor@ijsab.com Website: ijsab.com}




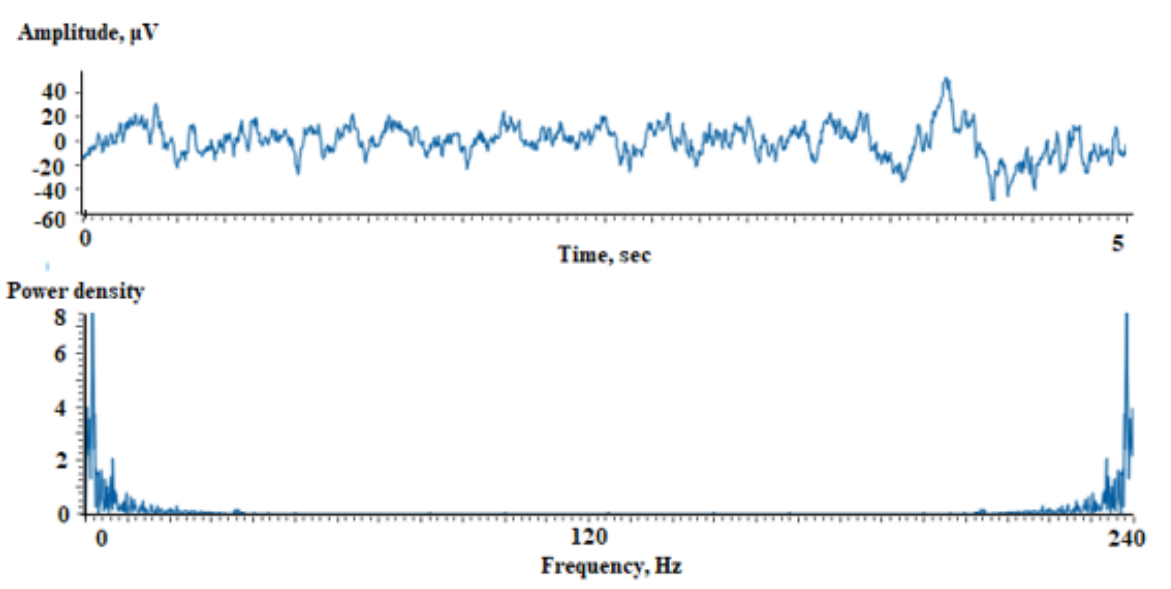

Fig.3. The implementation of the Fast Fourier transform, up - before, down - after implementing the Fourier transform

The disadvantages of this method are the fact that when Fourier decomposition, even for one given frequency, requires knowledge of the signal from the past and the future. Moreover, under conditions of the almost inevitable limitation of the number of harmonics or the spectrum of oscillations, an exact reconstruction of the signal after the direct and inverse Fourier transforms is theoretically impossible, due to the appearance of the Gibbs effect. As a result, online processing of brain signals carries difficulties.

Small discontinuities in a sinusoidal or any smoothly changing signal are difficult to detect in the Fourier spectrum, because they create many higher harmonics of very small amplitude. The spectrum of such signals contains subtle high-frequency components of the spectrum by which it is impossible to recognize the local peculiarity of the signal, and even more so its place and character.

\subsection{EEG wavelet transform}

Khan et al. (2020) and Balasubramanian et al. (2018) and Khalaf et al. (2019) and Kaleem (2018) proved that the fast Fourier transform can be used with high accuracy in conjunction with the wavelet transform.

The wavelet transform carries a huge amount of information about the signal, but, on the other hand, has a strong redundancy, since each point of the phase plane affects its result.

A continuous wavelet transform is defined as the scalar product of the original signal $\mathrm{x}(\mathrm{t})$ and the daughter wavelet function $\Psi_{t, a}(t)$ :

$W(\tau, a)=\left(x(t), \Psi_{\tau, a}(t)\right)=\int_{-\infty}^{+\infty} x(t) ¥ t_{\tau, a}^{*}(t) d t, \tau \in R^{+}$,

where $\mathrm{W}(\tau, \mathrm{a})$ - wavelet expansion coefficients; $\tau$, a - прarameters of time shift and scale, respectively; operator* means complex pairing.

Child wavelet functions $¥_{\tau, a}$, formed by shear and scale operations of the mother wavelet function $¥_{\tau}$ and related to it by the ratio:

$¥_{\tau, a}(t)=\frac{1}{\sqrt{a}} ¥\left(\frac{t-\tau}{a}\right)$

The complex Morlet wavelet, which is the product of a complex sinusoid and a Gaussian, is used as the mother wavelet function. The analytical expression of the Morlet wavelet has the form:

$¥(t)=\frac{1}{\sqrt[4]{\sigma^{2} * \pi}} * e^{-\frac{t^{2}}{2 \sigma^{2}}} * e^{j w_{0} t}$,

where $\omega 0$ - maternal wavelet center frequency; $\sigma$ - standard deviation of the envelope of the mother wavelet.

\section{International Journal of Science and Business} Email: editor@ijsab.com Website: ijsab.com 
Figure 4 shows the implementation of the wavelet transform by Bajaj (2020).

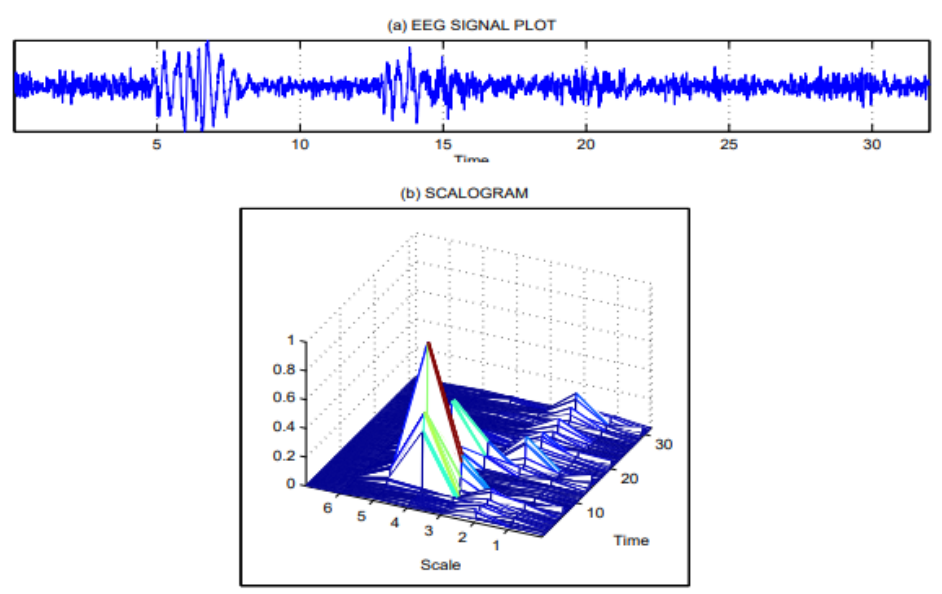

Fig. 4. Presentation of (up) a part of a one-channel EEG signal plot and the result of its analysis (down) by the wavelet transform based on harmonic wavelet functions and by the short-time Fourier transform

For wavelet transform it is especially important to remove noise from the signal before analyzing them. Wavelets seem to be a very convenient and promising mechanism for cleaning and pre-processing data for EEG signals. It is worth noting the following work in which the methods described above were involved. Salabun (2014) showed how the fast Fourier transform (FFT) is used to find the frequency components in a time-domain signal. Based on a sample signal and spectral analysis, the main EEG waves are presented. This document introduces how to record and process. EEG signal from MindWave MW001 in MATLAB environment. For this purpose, Fourier analysis has been used as an effective way to do spectral analysis. Shaker (2007) presented the classification of EEG waves, which is achieved using a discrete wavelet transform DWT with fast Fourier transform (FFT) by accepting normalized EEG data. The proposed method makes use of both a discrete wavelet transforms and a discrete Fourier transform. Especially, the wavelet transform is used as a classifier of EEG frequencies. Also, the filtered EEG data used as input for the wavelet transform offers ideal success in rejecting unwanted frequencies and allows DWT levels to distinguish between EEG waves only. Salabun (2019) showed that for a multi-stage process is used for preliminary processing of EEG data. Data is first pre-processed using Digital Signal Processing (DSP) filtering techniques to limit the effects of noise and artifacts, on the one hand, and determine the appropriate channels to be used on the other. Pre-processed signals are further analyzed using Fast Fourier Transform (FFT), Principal Component Analysis (PCA) for feature extraction, and k-nearest neighbor (KNN) for classification. Results for each step were presented and performance was evaluated. Dash (2014) demonstrated that for the analysis of epilepsy, the effective extraction of characters occurs due to Fourier and wavelet transforms. Delta, theta, alpha, beta, and gamma EEG sub-bands were obtained and studied to detect seizures and epilepsy. The extracted object is then applied to the ANN to classify the EEG signals. The authors concluded that the wavelet transform is a suitable method for frequency analysis of EEG for the analysis of epilepsy. Sellami et al. (2014), used a short-term Fourier transform (STFT) in the search for signs for identifying autism. Sawant et al. in the paper (2010), showed that EEG can be extracted by decomposing data using a multi-level discrete wavelet transform (DWT). Kocadagli et al. (2017) and Chatterjee et al.(2019) presented a general description of the work and methodologies for analyzing EEG signals in

\section{International Journal of Science and Business} Email: editor@ijsab.com Website: ijsab.com 
the time and frequency domains using the short-term FourierChin-Teng (2008) showed that EEG signals are sampled and then converted by three different feature extraction methods, including non-parametric weighted feature extraction (NWFE), principal component analysis (PCA), and linear discriminant analysis (LDA). Experimental data showed that NWFE + NBC gives the best classification accuracy in the range from $71 \%$ to $77 \%$, which is $10 \%-24 \%$ higher than LDA + KNN1. Lung (2015) devoted the article to the use of discrete wavelet transforms (DWT) in extracting a feature from an EEG signal obtained with a sensory response in children with autism. Training on a neural network with dedicated capabilities shows DWT that the network can achieve accuracy - 92.3\%. Nava (2017), presented three different schemes for extracting characteristics from an EEG signal: relative energy of the spectral band, harmonic parameters, and Takura distance. Spectral estimation is performed using autoregressive (AR) modeling. The authors compared the effectiveness of these schemes in order to select the optimal set of functions for a specific, sensitive and accurate neuro-fuzzy classification of sleep stages. The distance and central frequency of Takura seem to be the most promising for classifying the stages of sleep. Kołodziej (2011) and Umale et al. (2016) presented a new method for extracting features from the EEG signal for designing the brain-computer interface. The proposed algorithms are based on wavelet transform and higher-order statistics (HOS).

\subsection{Principal component method}

The method of principal components is a multidimensional statistical analysis technique used to reduce the dimension of the feature space with minimal loss of useful information. The potentials of Electrooculography (EOG) are one of the most popular artifacts that occur when the pupils move. In this case, the maximum amplitude of artifacts is observed in the frontal leads and decreases towards the occipital leads. An important point is that the EOG artifacts are not related to the current rhythm on the EEG and therefore they must be removed from the signal. To remove artifacts caused by involuntary eye movements of the subject from a multi-channel EEG, a wide analysis of the main components is used. Siuly et al. (2015) and Artoni et al. (2018) and Dea et al. (2019) and Polat et al. (2008) and Putilov et al. (2015) described this process in detail. The method of principal components consists of finding for the initial data such orthogonal transformation into a new coordinate system for which the following conditions are satisfied:

- selective dispersion of data along the first coordinate is maximum;

- sample dispersion of data along the kth coordinate is maximum under the condition of orthogonality to the first $(\mathrm{k}-1)$ coordinates.

As a result, the directions of the basis vectors will be chosen in such a way that the covariance coefficient between the projections of the initial data set on different coordinate axes will ultimately be zero, which will eliminate the effect of pupil movement on the result. The implementation of this method is presented in figure 5 .

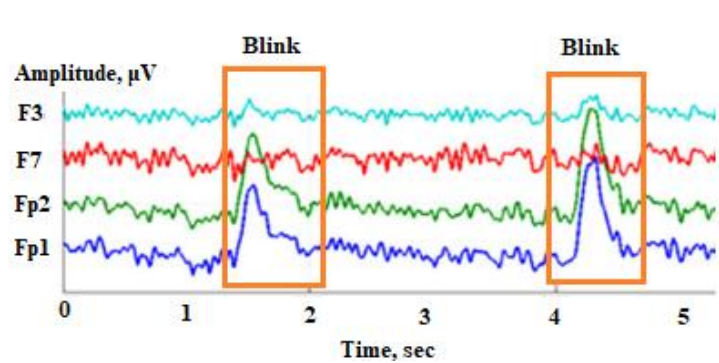

a

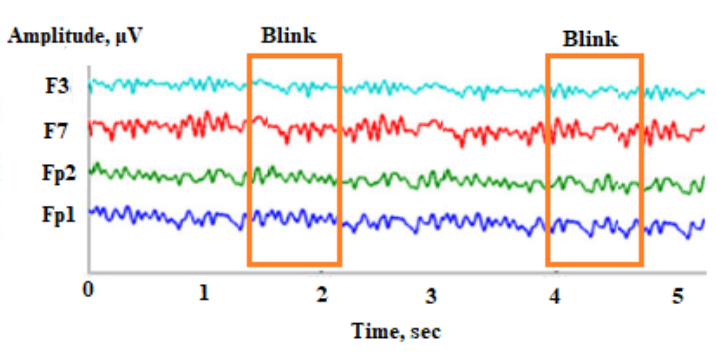

$\mathrm{b}$

\section{International Journal of Science and Business Email: editor@ijsab.com Website: ijsab.com}


Fig. 5. An example of removing artifacts from an EEG: a - recording of an electrooculogram; b reconstructed EEG signal without artifact component

For correct research in the field of classification of EEG signals, the electrooculogram potentials should be considered, because the neural network can take this artifact as a useful signal.

\subsection{General average method}

The method for measuring potential is when the potentials of the electrodes located on the surface of the head are measured concerning the potential of the electrode mounted on the earlobe.

The potential of the reference electrode mustn't change over time. But the conductivity of the scalp is electrically active, which leads to a measurement of potential. One of the simplest approaches to solving this problem is to recalculate the EEG signal values relative to the total averaged referent.

This calculation is performed according to the formula:

$\tilde{X}_{i}=X_{i}-\frac{1}{M} \sum_{i=1}^{M} X_{i}$,

where Xi is the rows of the matrix X (). In the ideal case, if the electrodes were uniformly located on the sphere, the total average for would be zero and the transition performed by expression (8) would allow restoring the exact values of the potentials.

At the same time, despite the importance of this operation, the mention of the Common averaged installation was found to be found in only a few works by Gazit et al. (2016) and Guerra et al. (2018) and Li et al. (2019) and Deguire et al. (2019).

\subsection{Other methods}

Several studies offer non-standard solutions for extracting traits from a signal. Singh et al. (2016) proposed a new model of feature detection based on the Hilbert - Huang transform, multidimensional empirical mode decomposition (MEMD), and Hilbert transform (HT). Automatically, comparisons are made with the expansion of only the Fourier series and only on one model of the classification neural network. Cic (2019) created three combined characteristics by combining spectral characteristics and time-frequency characteristics. The first combined function was a combination of all three types of functions used: RSD, IMFmed, and IMFch. The second combination was RSD and IMFmed, and the third combination was RSD and IMFch. The criterion of efficiency was the comparison with the Fourier series. Chatterjee (2019) presented the expansion of the EEG signal by a Fourier - Bessel series and the aperiodic Bessel functions. There are too many initial conditions for the EEG signal, which makes it difficult to use this method in practice.

Alomari (2013) proposed an automated computer platform for classifying electroencephalography (EEG) signals associated with movements of the left and right hands.. Data were pre-processed using EEGLAB MATLAB instrumentation and artifact removal was performed using AAR. Some works in an attempt to combine randomly use several methods. Ruddy et al. (2018) and Li et al. (2019) and Jain et al. (2019) and Übeyli et al. (2008) presented the results which only slightly surpass the results obtained by spectral Fourier analysis.

\section{Conclusion}

The authors are presented with the following algorithm for feature extraction from EEG signals. The algorithm includes the minimum operations necessary for the competent feature extraction from the signal, figure 6. Authors of EEG research are encouraged to use the

\section{International Journal of Science and Business Email: editor@ijsab.com Website: ijsab.com}


algorithm presented above to identify signs in EEG signals or, mention the reasons why these methods were not used. Analysis of the research on the topic of identifying signs with an EEG signal is complicated by the fact that there are no standards on this topic. Because of this, it is still quite difficult to conclude how successful the authors carried out feature extraction.

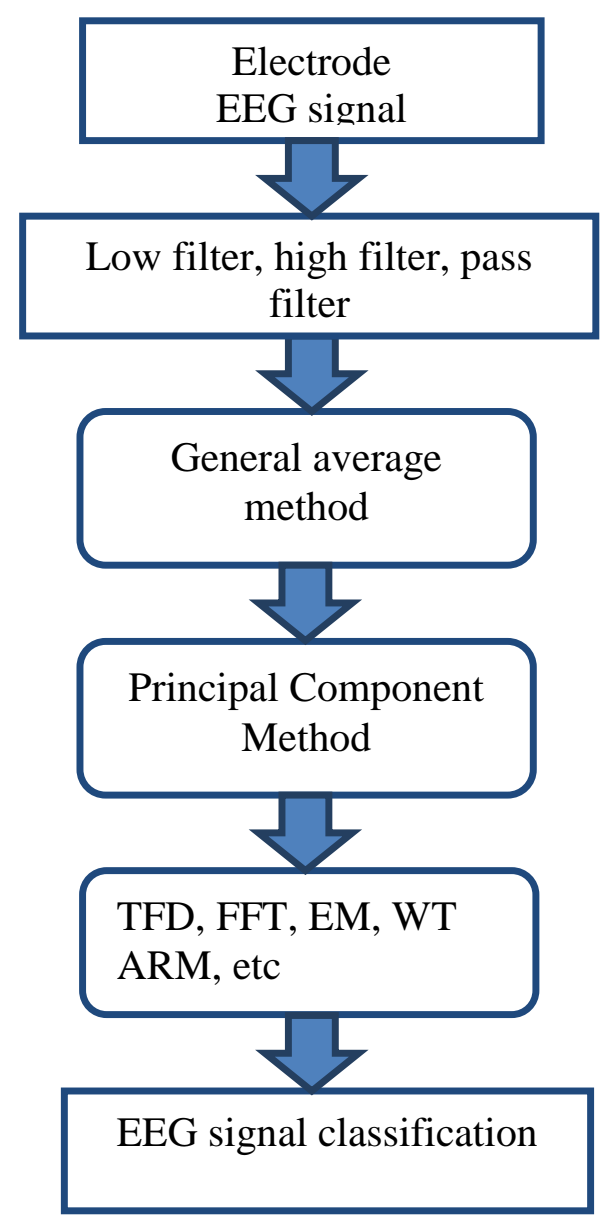

Fig. 6. Algorithm for feature extraction of EEG signals

In all works, the criterion for the success of extraction is the results that were obtained using the classification, which, according to the author of this work, is not correct. For example, each neural network model evaluates data in its way, and some models may not notice noise in the signal, but this does not mean the process of extracting feature was made correct. Since other models, with proper extraction, could show a higher percentage of correct answers during classification. To compare the correctness of the extraction of features from the signal, the authors often make comparisons with the fast Fourier method, the success criterion being the result of the accuracy of the classification model. At the same time, the fast Fourier series, although popular, is not a standard in the field of extraction by a sign from an EEG signal.

\section{References}

Al-Fahoum, A. (2014). Methods of EEG Signal Features Extraction Using LinearAnalysis in Frequency and Time-Frequency Domains. SRN Neuroscience, 7, 14-19

\section{International Journal of Science and Business Email: editor@ijsab.com Website: ijsab.com}


Alomari, M., Samaha, A., \& Kamha, K. (2013). Automated Classification of L/R Hand Movement EEG Signals using Advanced Feature Extraction and Machine Learning. (IJACSA) International Journal of Advanced Computer Science and Applications, 4, 207-212

Al-Salman, W., Li,Y. \& Wen, P. (2019). Detecting sleep spindles in EEGs using wavelet fourier analysis and statistical features. Biomedical Signal Processing and Control, 48, 80-92

Artoni, F., Delorme, A., \& Makeig, S. (2018). Applying dimension reduction to EEG data by Principal Component Analysis reduces the quality of its subsequent Independent Component decomposition. NeuroImage, 175, 176-187

Bajaj, N., Carrión, J., \& Berta, R. (2020). Automatic and tunable algorithm for EEG artifact removal using wavelet decomposition with applications in predictive modeling during auditory tasks. Biomedical Signal Processing and Control, 55, 224-230

Balasubramanian, G., Kanagasabai, A., \& Mohan, J. (2018). Music induced emotion using wavelet packet decomposition-An EEG study. Biomedical Signal Processing and Control, 42, 115-128

Chatterjee, R., \& Maitra, T. (2019). A novel machine learning based feature selection for motor imagery EEG signal classification in Internet of medical things environment. Future Generation Computer Systems, 98, 419-434

Chen, G. (2014). Automatic EEG seizure detection using dual-tree complex wavelet-Fourier features. Expert Systems with Applications, 41, Issue 5, 2391-2394

Chin-Teng, L. (2008). Research Article Nonparametric Single-Trial EEG Feature Extraction and Classification of Driver's Cognitive Responses. EURASIP Journal on Advances in Signal Processing, 7, 10-15

Cic, M. (2019). Optimal set of EEG features in infant sleep stage classification. Turkish Journal of Electrical Engineering \& Computer Sciences, 27, 605-615

Dash, D. (2014). Advanced Signal Processing Techniqes to Study Normal and Epileptic EEG. Conference: 41 st annual conference of OMS and International Conference on Industrial Mathematics and Scientific Computing At: India, ICIMSC14/OMS41

Dea, F., \& Stecca, M. (2019). A Big-Data-Analytics Framework for Supporting Classification of ADHD and Healthy Children via Principal Component Analysis of EEG Sleep Spindles Power Spectra. Procedia Computer Science, 159, 1584-1590

Deguire, F. (2019). The relationship between acute stress and EEG repetition suppression in infants. Psychoneuroendocrinology, 104, 203-209

Gazit, T. (2016). Dual array EEG-fMRI: An approach for motion artifact suppression in EEG recorded simultaneously with fMRI. NeuroImage, 142, 674-686

Guerra, A. (2018). TRIMAGE: A dedicated trimodality (PET/MR/EEG) imaging tool for schizophrenia. European Psychiatry, 50, 7-20

Hyvärinen, A., Ramkumar, P., Parkkonen, L., \& Hari, R. (2010). Independent component analysis of short-time Fourier transforms for spontaneous EEG/MEG analysis. NeuroImage, 49, Issue 1, 257-271

Kaleem, M., Guergachi, A., \& Krishnan, S. (2018). Patient-specific seizure detection in long-term EEG using wavelet decomposition. Biomedical Signal Processing and Control, 46, 157-165

Kato, K., Takahashi, K., Nobuaki, M., \& Ushiba, J. (2018). Online detection of amplitude modulation of motor-related EEG desynchronization using a lock-in amplifier: Comparison with a fast Fourier transform, a continuous wavelet transform, and an autoregressive algorithm. Journal of Neuroscience Methods, 293, 289-298

Khalaf, A., Sejdic, E., \& Akcakaya, M. (2019). Common spatial pattern and wavelet decomposition for motor imagery EEG- fTCD brain-computer interface. Journal of Neuroscience Methods, 320, 15, 98-106

Khan, H., Shanir, P., \& Farooq, O. (2020). A hybrid Local Binary Pattern and wavelets based approach for EEG classification for diagnosing epilepsy. Expert Systems with Applications, 140, 115-120

Kocadagli, O., \& Langari, R. (2017). Classification of EEG signals for epileptic seizures using hybrid artificial neural networks based wavelet transforms and fuzzy relations. Expert Systems with Applications, 88, 419-434 
Kołodziej, M. (2011). A New Method of EEG Classification for BCI with Feature Extraction Based on Higher Order Statistics of Wavelet Components and Selection with Genetic Algorithms, Conference: 2018 19th International Conference "Computational Problems of Electrical Engineering" (CPEE), 280-289

Li, H., Wang, D., Chen, J., \& Luo, X. (2019). Pre-service fatigue screening for construction workers through wearable EEG-based signal spectral analysis. Automation in Construction, 106, 340345

Li, X., Fan,H., \& Wang, L. (2019). Common spatial patterns combined with phase synchronization information for classification of EEG signals. Biomedical Signal Processing and Control, 52, 248256

Lung, C. (2015). Feature extraction of EEG signal using wavelet transform for autism classification, Journal of Engineering and Applied Sciences. 19, 8533-8540

Mane, A., Biradar, S., \& Shastri, K. (2019). Review paper on Feature Extraction Methods for EEG Signal Analysis. International Journal of Emerging Trend in Engineering and Basic Sciences, 2349-6967

Nava, P. (2017). EEG feature extraction for classification of sleep stages. Proceedings of the 26th Annual International Conference of the IEEE EMBS, 196-199

Polat, K., \& Güneș, S. (2008). Artificial immune recognition system with fuzzy resource allocation mechanism classifier, principal component analysis and FFT method based new hybrid automated identification system for classification of EEG signals. Expert Systems with Applications, 34, Issue 3, 2039-2048

Putilov , A. (2015). Principal component analysis of the EEG spectrum can provide yes-or-no criteria for demarcation of boundaries between NREM sleep stages. Sleep Science, 8, Issue 1, 16-23

Raut, A. (2010). EEG feature selection using mutual information and support vector machine: A comparative analysis. 32nd Annual International Conference of the IEEE EMBS Buenos Aires, Argentina, 4946-4947

Ruddy, K., Woolley, D., \& Mantini, D. (2018). Improving the quality of combined EEG-TMS neural recordings: Introducing the coil spacer. Journal of Neuroscience Methods, 294, 34-39

Salabun, A. (2019). Analysis of speech related EEG signals using emotive headset, fast fourier transform, principal component analysis, and K-nearest neighbors methods. Int J Biosen Bioelectron, 5(3), 94-98

Salabun, W. (2014). Processing and spectral analysis of the raw EEG signal from the MindWave. PRZEGLĄD ELEKTROTECHNICZNY, 5, 45-60

Sawant, H., \& Jalali, Z. (2010). Detection and classification of EEG waves. Oriental Journal of Computer Science \& Technology, 3(1), 207-213

Sellami, L., \& Neubig, T. (2014). EEG Interpretation through Short Time Fourier Transform for Sensory Response Among Children, Australian Journal of Basic and Applied Sciences, 8(5), 417422

Shaker, M. (2007). EEG Waves Classifier using Wavelet Transform and Fourier Transform. International Journal of Medical, Health, Biomedical, Bioengineering and Pharmaceutical Engineering, 3, 220-225

Singh, M., \& Goyat, R. (2016). Feature Extraction for the Analysis of Multi-Channel EEG Signals Using HilbertHuang Technique. International Journal of Engineering and Technology, 8, 17-27

Sitnikova, E., Hramov, A., Koronovsky, A., \& Luijtelaar, G. (2009). Sleep spindles and spike-wave discharges in EEG: Their generic features, similarities and distinctions disclosed with Fourier transform and continuous wavelet analysis. Journal of Neuroscience, 180, Issue 2, 304-316

Siuly, S., \& Li,Y. (2015). Designing a robust feature extraction method based on optimum allocation and principal component ana. Computer Methods and Programs in Biomedicine, 119, Issue 1, 29 42

Übeyli, E. (2008). Analysis of EEG signals by combining eigenvector methods and multiclass support vector machines. Computers in Biology and Medicine, 38, Issue 1, 14-22

\section{International Journal of Science and Business Email: editor@ijsab.com Website: ijsab.com}


Umale, C., Vaidya, A., \& Shirude, S. (2016). Feature Extraction Techniques and Classification Algorithms for EEG Signals to detect Human Stress - A Review. International Journal of Computer Applications Technology and Research, 5, Issue 1, 08 - 14, 8-14

V.Jain, S., Dye, T., \& Kedia, P. (2019). Value of combined video EEG and polysomnography in clinical management of children with epilepsy and daytime or nocturnal spells. Seizure, 65, 1-5

\section{Cite this article:}

Rakhmatulin Ildar (2020). Review of EEG feature selection by neural networks. International Journal of Science and Business, 4(9), 101-112. doi: https://doi.org/10.5281/zenodo.3987894

Retrieved from http://ijsab.com/wp-content/uploads/592.pdf

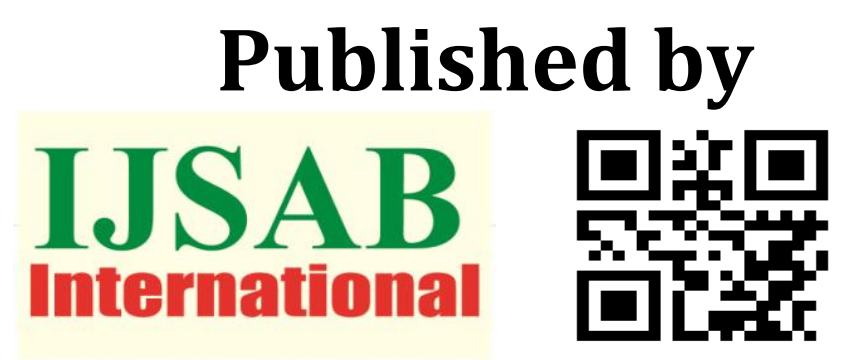

